\title{
XXII.
}

\section{Ueber einige Veränderungen, welche das Quecksilber im thierischen Organismus hervorruft.}

Von Dr. Sajkowsky aus Moscau.

Die Wirkung der Heilmittel betrifft nach deren Verbreitung im Körper vorzugsweise einzelne Organe oder Organtheile, daher auch bestimmte Heilmittel der Erfabrung gemäss gewisse Arten und Gruppen von Symptomen (wie sie dem einen oder anderen Organ eigen sind) hervorrufen. Diese Thatsache ist seit den ältesten Zeiten bekannt. Sie gab schon den alten Aerzten Veranlassung, die Mittel nach dieser Beziehung zu benennen: Brust-, Hirn-, Lebermittel u. s. w. Man hat diese Wirkungsweise der Heilmittel als specifisch betrachtet und nannte sie desshalb "Specifica localia“ (Rademacher's Organheilmittel). Hat sich die Wissenschaft erst vieler solcher Mittel bemächtigt, welche, wie das Kali und Natron (Virchow) auf die Zellen?des Flimmerepithels, einen specifischen und directen Einfluss auf das Eigenleben der verschiedenen, die specifischen Organe bildenden Zellenarten üben, so wird dadurch dem Arzt eine entschiedene Macht über die Lebensäusserungen derselben gegeben und dann wird die Medicin im Stande sein, Heilwirkungen $\mathrm{zu}$ veranlassen.

So eine specifische Wirkung lässt sich auch am Quecksilber demonstriren. Wir haben meistentheils an Kaninchen experimentirt und haben fast constant eine und dieselbe Wirkung beobachtet. In den meisten Fällen wurde das Quecksilberchlorid $(\mathrm{Hg} \mathrm{Cl})$ angewandt; dasselbe bekamen die Thiere in wässeriger Lösung, manchmal innerlich, meistentheils subcutan; constant wirkte die subcutan injicirte Dosis viel intensiver und schneller als die innerlich gebrauchte und an Kaninchen in deu folgenden Verhältnissen: $1: 12-15$, d. h. nach einer Dosis von 0,04-0,06 Grm. in 2 Tagen subcutan starben die Thiere nach 3 Tagen mit den charakteristischen Veränderungen in den Organen, während von 2 eben so grossen Kaninchen, die 0,03-0,05 Grm. täglich innerlich bekamen, 
das eine nach 12, das andere nach 16 Tagen starb und die später zu beschreibenden Veränderungen in höchst unbedeutendem Maasse zeigten.

An Thieren, die das $\mathrm{HgCl}$ subcutan bekamen, wurde constant eine mehr oder weniger starke Diarrhoe beobachtet und bei der Section solcher Thiere wurde ohne Ausnahme eine sehr intensive Hyperämie der Mucosa der Gedärme und manchmal sehr zahlreiche, hämorrhagische Heerde im Magen beobachtet. Die Excremente enthielten sehr deutliche Spuren von Hg, das electrolytisch nach Schneider's Methode*) (mit J), nach vorhergegangener Zerstörung der organischen Substanzen mit $\mathrm{HCl}$ und chlorsaurem Kali nachgewiesen wurde. Dass man auf eine Ausscheidung des $\mathrm{Hg}$ in das Darmlumen allein nach dem Gesagten schliessen darf, können wir nicht behaupten, denn wir haben an Hunden (in 3 Fällen $2 \mathrm{Mal}$ ), denen wir, nach 2 tägigem subcutanen Gebrauch von 0,05 bis $0,1 \mathrm{Grm}$. Sublimat, eine gläserne Canüle in den Kanal der Submaxillardrüse eingeführt, und durch wechselnde Reizung der Chorda tymp. und des Sympathicus gegen $\frac{1}{2}$ Unze Speichel entzogen hatten, in diesem Speichel oft so beträchtliche Mengen von $\mathrm{Hg}$ angetroffen, dass wir im Stande waren, dasselbe krystallinisch als Jodverbindung darzustellen. Es bleibt also die Möglichkeit offen, dass das gefundene Hg zum Theil dem verschluckten Speichel angehörte.

Was nun die ferneren pathologischen Veränderungen anbetriff, die man bei der Autopsie solcher Thiere findet, so zeigt die Untersuchung aller Bauchorgane eine sehr bedeutende Gefässerweiterung, besonders in den Nieren und in der Leber (vorzüglich an Kaninchen). Ausserdem beobachtet man bei letzteren noch, ohne Ausnahme, nach subcutanen Einspritzungen von $0,02-0,04 \mathrm{Grm} . \mathrm{HgCl}$ schon nach $18-28$, Stunden eine beträchtliche Ablagerung von Salzen in den gestreckten Harnkanälchen der Rindensubstanz; diese Ablagerung wird nach und nach immer intensiver, so dass nach 2 Tagen solche Nieren mit den genannten Massen wie vollgepfropft erscheinen. Diese pathologische Ablagerung kommt ausnahmslos nur in der genannten Region der Nieren vor. Mit blossem Auge kann man dieselbe sehr deutlich beobachten, indem ein Längs-

*) Schneider, Sitz.-Bericht d. Natur-Mathen. Classe d. Wiener Akad. der Wissensch. Bd. 40. S. 239. 1860. 
schnitt durch die ganze Niere mit weissen, derben Streifen wie durchzogen erscheint.

Gewöhnlich verschafften wir uns vor der ersten Injection ein Probestückchen der gesunden Niere des Thieres. Die Niere wurde mit den Fingern fest an die Bauchwand gedrückt, die Haut gespalten, ein kleines Stück der Rindensubstanz ausgeschnitten und unter dem Mikroskop untersucht, wobei das Bild einer ganz normalen Structur hervortrat. Dann wurde die Injection gemacht, und nach 18-28 Stunden eine $z$ weite Probe geholt; die mikroskopische Untersuchung zeigte schon eine beträchtliche Ablagerung im Epithel, welche bis zum Tode des Thieres an Volumen zunahm. Die in den Harnkanälchen abgelagerte Substanz stellt sich als amorphe, mehr oder weniger stark glänzende, stark das Licht reflectirende, gleichförmige Masse dar, welche das Lumen des Harnkanälchens so vollstopft, dass man nicht im stande ist, sein Epithel zu unterscheiden. Diese Masse ist sowohl in kaltem, als auch in heissem, sogar kochendem Alkohol und Aether unlöslich, löst sich aber sehr leicht in verdünnten Mineralsäuren (Schwefelsäure, HCl), indem unter ziemlich unbedeutender Gasentwickelung die dunklen Massen verschwinden, das Feld des objects klar wird, an den afficirten Stellen das Epithel wieder erscheint und eine mehr oder weniger bedeutende Quantität von sternförmigen Krystallen sich bildet. Die chemische Untersuchung solcher Ablagerungen wies bedeutende Mengen phosphorsauren Kalk nach, welcher, soweit es sich bis jetzt aus den Reactionen schliessen lässt, die Hauptmasse solcher Ablagerungen bildet; daneben wurden auch kohlensaurer Kalk in viel geringerer Menge und Spuren von Kochsalz constatirt.

Bei Hunden findet man dieselbe Localität der Niere angegriffen, aber durch einen ganz anderen Prozess. An solchen mit Sublimat subcutan vergifteten Thieren sieht man oft in den gestreckten Harnkanälchen der Corticalsubstanz eine mehr oder weniger (je nach Zeit und Dosis) intensive Fettdegeneration des Epithels derselben, sehr oft findet man anstatt normaler Kanälchen ganz wie mit einer Emulsion gefüllte Schläuche. $(\mathrm{Ob}$ das ein zufälliger Befund sei - wir baben im Ganzen die Nieren von nur 3 Hunden untersucht - wollen wir als offne Frage dahingestellt sein lassen, und zwar um so mehr, als auch Prof. Rindfleisch neuerdings in seinem Lehrbuche (S. 22) die Beobachtung mittheilt, dass man bei 
Hunden unter normalen Verhältnissen solche Veränderungen des Nierenepithels antrifft)*).

Was aber eigenthümlich in allen beschriebenen Fällen ist, das ist die Ungleichmässigkeit der Verbreitung des Prozesses: die gestreckten Kanälchen der Rindensubstanz werden nie alle gleichmässig, sowohl bei Kaninchen, als auch bei Hunden angegriffen, sondern es breitet sich der Prozess wie mit Sprüngen aus, indem nur eine gewisse Menge (wie Paquete von $3-5$ Harnkanälchen) angegriffen erscheint, und zwischen den afficirten Theilen mitunter ganz normale liegen, so dass auf diese Weise eine solche Niere eine gestreifte Zeichnung erhält. Dieser Befund scheint gegen eine vollkommene Identität der Function des Epithels der gestreckten Harnkanälchen der Rindensubstanz zu sprechen.

Ausser dieser Veränderung beobachtet man noch eine eigenthümliche Erscheinung, die wir oft an Kaninchen wahrgenommen haben (unter 12 Fällen $8 \mathrm{Mal}$ ): die physikalischen Eigenschaften des Harns zeigen im Laufe der Zeit (bald schon am anderen Tage nach der ersten Injection, bald nach 2-3 Tagen nach verhältnissmässig stärkeren Dosen) folgende Veränderungen: der Harn wird in grösseren Mengen abgesondert, wird blass, durchsichtig, was man am normalen Kaninchenharn nie beobachtet. (Es scheint in den meisten Fällen diess Klarwerden des Harns Hand in Hand mit der Ablagerung der Kalksalze in den Harnkanälchen der Niere zu gehen.) Ausserdem enthält er mehr oder weniger bedeutende Mengen Zucker. Wir haben in 5 Fällen einen so starken Diabetes an solchen Thieren beobachtet, dass $2 \mathrm{Ccm}$. Harn bei der Gährung in 48 Stunden bei einer Temperatur von $31^{\circ} \mathrm{C}$. gegen $3-4 \mathrm{Ccm}$. $\mathrm{CO}_{2}$ gegeben haben. Wir müssen noch bemerken, dass dieser künstlich hervorgebrachte Diabetes manchmal 4-5 Tage dauert, ja selbst in einem Falle eine ganze Woche (nach der Dosis von $0,05 \mathrm{Grm}$. $\mathrm{HgCl}$ in $4-5 \mathrm{Mal}$ injicirt). Dabei starben die Thiere nicht. Diese Dauer ist die längste von allen bis jetzt von uns künstlich hervorgerufenen Diabetes-Dauern (siehe Centralblatt für medicin. Wissenschaften. 1865. No. 49). An 2 Hunden, die chro-

*) Ob die von Kussmaul (Untersuchungen über den constitutionellen Mercurialismus. S. 432) beim quecksilbervergifteten Mano beschriebenen Veränderungen der Niere mit unserem Befunde zusammen zu bringen seien, lässt sich für den Augenblick nicht entscheiden. 
nisch vergiftet wurden, indem ihnen subcutan sehr grosse Mengen $\mathrm{HgCl}$ während ganzer Wochen einverleibt wurden (zu 2 Gran 13 bis 18 Tage lang) und die dabei sehr abgemagert waren, haben wir ebenfalls einen äusserst starken Diabetes nach Verlauf von 4 Wochen beobachtet. (Beide Hunde gingen leider durch einen $\mathrm{Zu}$ fall während unsrer Abwesenheit zu Grunde.) Jedenfalls sind diese Fälle mit den von Kletzinsky am merkurialkranken Menschen zusammenzustellen *).

Indem wir nun einerseits eine so constante Erscheinung, wie die Ablagerung der Kalksalze in den gestreckten Harnkanälchen der Rindensubstanz und das Erscheinen des Diabetes an mit $\mathrm{Hg} \mathrm{Cl}$ subcutan vergifteten Kaninchen vor uns hatten, glaubten wir andererseits ein festes Criterium zur Entscheidung der Frage, ob alle Quecksilberpräparate auf eine und dieselbe Weise auf den Organismus ihre Wirkung ausüben, gefunden zu haben. $\mathrm{Zu}$ diesem Zwecke wandten wir Calomel und Jodquecksilberverbindungen, das Deutojoduret. hydrarg., als das mehr lösliche, an. Wir verrieben das $\mathrm{Hg}_{2} \mathrm{Cl}$ und $\mathrm{HgJ}$ mit Glycerin und injicirten unter die Haut. Calomel wurde $\mathrm{zu} 5$ Gran 2-3 Tage eingespritzt, wovon am 4ten Tage die Thiere zu Grunde gingen, indem sie bei Lebzeiten einen sehr intensiven Diabetes und post mortem dieselben pathologischen Ablagerungen in den gestreckten Harnkanälchen der Rindensubstanz darboten.

Das Jodquecksilber brachte einen sehr stark ausgesprochenen Diabetes hervor, der auch 2-4 Tage dauerte; die Ablagerung der Kalksalze aber war sehr unbedeutend.

Bei diesen Versuchen stiessen wir auf folgende Eigenthümlichkeit: indem wir die Wirkung des Sublimats und Calomels auf die Mäuse untersuchten (sie bekamen eine Paste aus Eigelb, Mehl, Zucker und $\mathrm{Hg}_{2} \mathrm{Cl}$ oder $\mathrm{HgCl}$ ), stellte sich heraus, dass solche Thiere bloss eine minimale Dose $\left(\frac{1}{60}-\frac{1}{45} \mathrm{Grn}\right.$.) Calomel zu verschlucken brauchten, um nach $2-3$ Stunden paralysirt zu werden und zu Grunde zu geben. Eine viel bedeutendere Menge Sublimat (Grn. $\frac{1}{T^{\prime}}$ ) rief dieselbe Erscheinung erst nach Verlauf von $1 \frac{1}{2}-$ 2-5 Tagen hervor.

*) Kussmaul, Untersuchungen über den constit. Mercurialismus, S. 244. 